\title{
OIL GLANDS NUMBER AND OIL GLANDS DIAMETERS OF Thymbra spicata
} var. spicata L. LEAVES

\author{
DURMUŞ ALPASLAN KAYA*, FILIZ AYANOĞLU \\ Mustafa Kemal University, Faculty of Agriculture, Department of Field Crops, \\ dak1976@msn.com
}

\begin{abstract}
In the study, a genetic pool was created with plant samples taken from the locations where the Thymbra spicata var. spicata L. plant, which is culturally and economically important, grows densely in the province of Hatay. Plants were propagated with cuttings taken from these single plants. 213 plants were collected from 68 different locations for the genetic pool. The leaves of the plants in this gene pool were examined in terms of the number of essential oil glands per unit area and the diameter of the essential oil glands. The number of glands per unit area in plant leaves showed a wide variation and ranged from 5.61 to 56.04 pieces $/ \mathrm{mm}^{2}$. The diameters of the oil glands varied between $75.40-112.86 \mu \mathrm{m}$ and the average diameter was determined as $94.09 \mu \mathrm{m}$ In the study, it was determined that some plants with low essential oil ratios such as Z144 and Z158 also have low oil glands number and oil glands diameter. It would be appropriate to evaluate these values obtained as preliminary knowledge together with future ontogenetic variability studies.
\end{abstract}

Keywords: Thymbra spicata var. spicata L, essential oil gland.

\section{INTRODUCTION}

The plant species that make up plant formations do not show the same characteristics everywhere. With the emergence of local differences arising from climate, soil and geomorphological features, the plant species that make up the plant communities diversify. The region, which includes many mountains, plateaus and valley areas, especially Amanos mountains, is in a very rich position in terms of biological resources. In addition, there is a rich biological diversity with the amount of rainfall and high relative humidity in the summer months (Çakan and Byfield, 2005; Vural and Aytaç 2005; Avc1, 2005). There are plant species that are naturally found in this region, almost all of which are collected from nature or traded for various purposes. Among these, Thymbra spicata var. spicata L. comes first.

Thyme species are most export products made of medicinal and aromatic plants in Turkey (Özgüven ve ark. 2005). Flora of Turkey has four species of this plant is located naturally which are Thymbra spicata var. spicata, Thymbra spicata var. intricata, Thymbra sintenisii var. sintenisii and Thymbra sintenisii var. isaurica (Davis, 1982; Başer, 2002). Thymbra spicata var. spicata L., which is called sater, zahter or blackhead thyme (Baytop, 1994) naturally grows intensively in the Eastern Mediterranean region, especially in the province of Hatay. The plants collected from nature are mostly consumed fresh traditionally in the region. In addition, it is dried and used as a spice and tea, and its essential oil, which is rich in carvacrol, is also used for different purposes. T. spicata $\mathrm{L}$. is a plant that naturally grows intensively in Mediterranean countries and in the Mediterranean region of Turkey, especially in Hatay province.

Although there are many studies on the morphology, anatomy, amount of essential oil, components and antimicrobial properties of the thymbra plant, studies on agriculture are very limited in Tukey (Doğan et al., 1987; Hancı et al., 2003; Özel et al., 2003; Baydar et al., 2004; Erken, 2005; Özcan et al., 2008). Inan et al. (2011), in their study on Thymbra spicata L., which they collected from Adiyaman region, determined that the amount of essential oil changed in different harvest times and that the highest rate of 
essential oil was obtained from plants harvested during the flowering period. Kiz1 (2013) made evaluations on 28 different populations collected from Southeast Anatolia and the Mediterranean region and determined that there were significant differences between the populations. Carvacrol is the most abundant component as the main component (Başer, 2002). But Hanc1 et al. (2003) stated that this species also has thymol-containing chemotypes.

Although standardization is important in all other agricultural products, it is even more important for medicinal plants. Because products that do not meet certain quality standards cannot be traded. Ensuring standardization in agricultural products is possible by growing standard products. This can only be achieved through variety breeding. Breeding studies are long-term but must be done. First of all, the genetic material we have should be well known and its genetic potentials should be revealed. In this study, the number of glands per unit area and the size of the oil glands in the leaves of the thymbra grown in different locations in the flora of Hatay, and the infrastructure for future single plant selection improvement. is intended to be created. For this purpose, a genetic pool was created for $T$. spicata L. plant with plant samples selected from different locations where the plants are densely grown and these plants were examined in terms of oil glands.

\section{MATERIALS AND METHODS}

Survey studies were carried out in areas where Thymbra spicata L. plant spread in Hatay province and collection studies were carried out in these areas. As a result of the studies 213 single plants were selected from 68 different locations and were examined in terms of the number of oil glands per unit area and the size of oil glands. Plants were selected primarily different, well developed and healthy ones according to the single plant selection method. The oil cells of the Thymbra spicata L. species are found in sessile glandular hairs (Davis, 1982). In the study, the number of oil glands and the size of oil glands for each individual plant were determined. Later, plants propagated with cuttings taken from these plants were planted in the collection garden.

\section{Number of Oil Glands per Unit Area $\left(\mathrm{pcs} / \mathrm{mm}^{2}\right)$}

JEOL brand JSM-5500LV model SEM (Scanning Electron Microscope, X 300.000) electron microscope was used at Science Research and Application Center in Mustafa Kemal University. In each sample, the number of oil glands per unit area on 4 leaves (4 replication) of the plant was determined in terms of units/mm2 and averaged. The largest middle part of the leaf samples was used in counting and measurements made in SEM.

\section{Average Oil Gland Diameter $(\mu \mathrm{m})$}

In the imaging performed to determine the number of oil glands per unit area, the diameter of 5 oil glands of these 4 leaves was determined for each plant and the average was obtained. A total of 20 measurements were made for each plant. 


\section{RESULTS AND DISCUSSION}

\section{Number of Oil Glands per Unit Area $\left(\mathrm{pcs} / \mathrm{mm}^{2}\right)$}

As a result of the counts and measurements made on the SEM (Scanning Electron Microscope) images on the leaves of thymbra plants collected from the flora of Hatay, the number of essential oil glands per $\mathrm{mm}^{2}$ was determined and given in Table 1 . The average number of oil glands of ecotypes in the experiment was $18.74 \mathrm{pcs} / \mathrm{mm}^{2}$. The lowest oil gland numbers were obtained from the ecotypes Z158 with $5.61 \mathrm{pcs} / \mathrm{mm}^{2}$ and Z144 with $5.89 \mathrm{pcs} / \mathrm{mm}^{2}$. The highest numbers of oil glands were obtained from ecotypes Z98 (56.04 $\left.\mathrm{pcs} / \mathrm{mm}^{2}\right), \mathrm{Z} 131\left(44.57 \mathrm{pcs} / \mathrm{mm}^{2}\right)$ and Z102 (41.59 $\left.\mathrm{pcs} / \mathrm{mm}^{2}\right)$ respectively.

Table 1. The number of oil glands of T. spicata var. spicata L. leaves (pcs $/ \mathrm{mm}^{2}$ )

\begin{tabular}{|c|c|c|c|c|c|c|c|c|c|}
\hline Code & Glands & Code & Glands & Code & Glands & Code & Glands & Code & Glands \\
\hline $\mathrm{Z1}$ & 20.23 & Z44 & 17.43 & Z87 & 14.76 & $\mathrm{Z} 130$ & 16.18 & Z173 & 16.67 \\
\hline $\mathrm{Z} 2$ & 9.26 & $\mathrm{Z} 45$ & 28.44 & Z88 & 16.17 & Z131 & 44.57 & Z174 & 21.04 \\
\hline $\mathrm{Z3}$ & 26.63 & Z46 & 11.13 & Z89 & 15.39 & Z132 & 9.81 & Z175 & 20.17 \\
\hline $\mathrm{Z} 4$ & 15.91 & Z47 & 16.32 & Z90 & 19.25 & $\mathrm{Z} 133$ & 15.90 & Z176 & 19.75 \\
\hline $\mathrm{Z} 5$ & 16.41 & $\mathrm{Z} 48$ & 15.87 & Z91 & 24.24 & Z134 & 10.09 & Z177 & 18.05 \\
\hline Z6 & 28.11 & Z49 & 16.57 & Z92 & 22.10 & Z135 & 20.31 & Z178 & 19.87 \\
\hline $\mathrm{Z7}$ & 22.63 & $\mathrm{Z} 50$ & 17.28 & Z93 & 21.69 & Z136 & 9.05 & Z179 & 21.07 \\
\hline Z8 & 16.57 & Z51 & 16.34 & Z94 & 22.33 & Z137 & 11.97 & Z180 & 20.19 \\
\hline Z9 & 7.82 & Z52 & 14.62 & Z95 & 28.08 & Z138 & 12.78 & Z181 & 18.74 \\
\hline $\mathrm{Z} 10$ & 17.13 & Z53 & 25.27 & Z96 & 23.79 & Z139 & 19.47 & Z182 & 20.14 \\
\hline $\mathrm{Z} 11$ & 19.46 & Z54 & 25.43 & Z97 & 31.10 & $\mathrm{Z} 140$ & 22.02 & Z183 & 20.78 \\
\hline $\mathrm{Z} 12$ & 17.88 & Z55 & 13.41 & Z98 & 56.04 & Z141 & 16.41 & Z184 & 19.84 \\
\hline $\mathrm{Z} 13$ & 12.46 & Z56 & 19.84 & Z99 & 14.98 & Z142 & 10.12 & Z185 & 18.96 \\
\hline $\mathrm{Z} 14$ & 18.66 & Z57 & 23.37 & $\mathrm{Z} 100$ & 15.47 & $\mathrm{Z} 143$ & 22.75 & Z186 & 19.32 \\
\hline $\mathrm{Z} 15$ & 17.34 & Z58 & 10.04 & Z101 & 16.32 & Z144 & 5.89 & Z187 & 21.04 \\
\hline $\mathrm{Z} 16$ & 12.26 & Z59 & 30.40 & Z102 & 41.59 & Z145 & 9.98 & Z188 & 20.78 \\
\hline $\mathrm{Z} 17$ & 19.32 & Z60 & 12.48 & Z103 & 9.65 & Z146 & 22.40 & Z189 & 19.43 \\
\hline $\mathrm{Z} 18$ & 15.24 & Z61 & 18.58 & $\mathrm{Z} 104$ & 28.38 & $\mathrm{Z} 147$ & 16.70 & Z190 & 17.54 \\
\hline Z19 & 12.92 & Z62 & 19.48 & Z105 & 15.87 & Z148 & 13.36 & Z191 & 16.35 \\
\hline $\mathrm{Z} 20$ & 15.32 & Z63 & 18.98 & Z106 & 11.99 & Z149 & 36.86 & Z192 & 19.03 \\
\hline $\mathrm{Z} 21$ & 24.98 & Z64 & 27.03 & Z107 & 12.56 & $\mathrm{Z} 150$ & 6.82 & Z193 & 16.79 \\
\hline $\mathrm{Z} 22$ & 14.20 & Z65 & 21.02 & Z108 & 9.62 & Z151 & 18.27 & Z194 & 21.08 \\
\hline $\mathrm{Z} 23$ & 20.10 & Z66 & 10.73 & Z109 & 23.16 & Z152 & 15.73 & Z195 & 22.05 \\
\hline Z24 & 14.78 & Z67 & 18.87 & Z110 & 14.36 & Z153 & 36.54 & Z196 & 19.56 \\
\hline $\mathrm{Z} 25$ & 10.30 & Z68 & 15.10 & Z111 & 10.55 & Z154 & 28.66 & Z197 & 18.96 \\
\hline Z26 & 20.53 & Z99 & 22.59 & $\mathrm{Z} 112$ & 10.96 & Z155 & 12.19 & Z198 & 18.61 \\
\hline $\mathrm{Z} 27$ & 19.12 & $\mathrm{Z70}$ & 16.54 & Z113 & 16.07 & Z156 & 22.14 & Z199 & 18.97 \\
\hline Z28 & 18.65 & Z71 & 17.02 & Z114 & 26.43 & Z157 & 19.78 & Z200 & 20.14 \\
\hline Z29 & 30.30 & Z72 & 21.75 & Z115 & 22.12 & Z158 & 5.61 & Z201 & 21.59 \\
\hline $\mathrm{Z} 30$ & 29.42 & Z73 & 20.74 & Z116 & 12.02 & Z159 & 19.28 & Z202 & 22.03 \\
\hline $\mathrm{Z} 31$ & 22.10 & Z74 & 19.18 & Z117 & 18.02 & Z160 & 10.88 & Z203 & 16.57 \\
\hline $\mathrm{Z} 32$ & 20.11 & Z75 & 16.84 & Z118 & 17.45 & Z161 & 18.11 & Z204 & 20.45 \\
\hline $\mathrm{Z} 33$ & 19.17 & Z76 & 10.12 & Z119 & 17.68 & Z162 & 19.81 & Z205 & 19.52 \\
\hline Z34 & 18.66 & Z77 & 19.19 & Z120 & 16.37 & Z163 & 22.14 & Z206 & 17.51 \\
\hline $\mathrm{Z} 35$ & 18.94 & Z78 & 21.05 & Z121 & 12.94 & Z164 & 21.94 & Z207 & 16.29 \\
\hline Z36 & 11.79 & Z79 & 20.12 & $\mathrm{Z} 122$ & 17.65 & Z165 & 17.89 & Z208 & 18.97 \\
\hline Z37 & 22.02 & Z80 & 19.01 & Z123 & 27.21 & Z166 & 6.34 & Z209 & 21.04 \\
\hline Z38 & 13.95 & Z81 & 11.73 & Z124 & 28.85 & Z167 & 8.21 & Z210 & 20.10 \\
\hline Z39 & 11.87 & Z82 & 17.92 & $\mathrm{Z} 125$ & 20.95 & Z168 & 19.62 & $\mathrm{Z} 211$ & 19.87 \\
\hline $\mathrm{Z} 40$ & 11.04 & Z83 & 24.57 & Z126 & 28.84 & Z169 & 15.79 & Z212 & 19.10 \\
\hline Z41 & 33.35 & Z84 & 12.49 & Z127 & 12.62 & $\mathrm{Z} 170$ & 18.04 & Z213 & 19.12 \\
\hline $\mathrm{Z} 42$ & 14.52 & Z85 & 21.15 & Z128 & 20.04 & Z171 & 17.93 & & \\
\hline $\mathrm{Z} 43$ & 30.91 & Z86 & 13.2 & Z129 & 19.87 & $\mathrm{Z} 172$ & 18.97 & & \\
\hline
\end{tabular}

Min: 5.61; Max: 56.04; Average: 18.74; Std. deviation: 6.45; Coef. of variation: 34,41

https://doi.org/10.24264/icams-2020.II.14 


\section{Average Oil Gland Diameter $(\mu \mathrm{m})$}

In the study, measurements made for each individual thymbra plant collected and the average diameter was determined as $\mu \mathrm{m}$ and given in Table 2 . The diameters of the oil glands of the collected thymbra varied between 75,40-112,86 $\mu \mathrm{m}$. The highest average diameter values were obtained from plants with code number Z17 $(112.86 \mu \mathrm{m})$, Z31 $(106.57 \mu \mathrm{m})$ and Z141 $(105.25 \mu \mathrm{m})$, respectively. In the experiment, the lowest mean diameter values were obtained from ecotypes Z158 $(75.40 \mu \mathrm{m}), \mathrm{Z} 52(80.29 \mu \mathrm{m})$, Z137 $(81.60 \mu \mathrm{m})$ and Z144 $(82.20 \mu \mathrm{m})$. The diameter of an oil glands in the thymbra leaves was determined to be 94.09 on average.

Table 2. The average oil gland diameters of $T$. spicata var. spicata L. leaves $(\mu \mathrm{m})$

\begin{tabular}{|c|c|c|c|c|c|c|c|c|c|}
\hline Code & Gland. & Code & Glands & Code & Glands & Code & Glands & Code & Glands \\
\hline $\mathrm{Z} 1$ & 99.75 & Z44 & 99.76 & Z87 & 92.22 & Z130 & 93.00 & Z173 & 104.86 \\
\hline $\mathrm{Z} 2$ & 88.44 & $\mathrm{Z} 45$ & 99.71 & Z88 & 101.75 & Z131 & 97.00 & Z174 & 87.80 \\
\hline $\mathrm{Z3}$ & 103.25 & Z46 & 96.22 & Z89 & 99.60 & Z132 & 88.22 & Z175 & 95.73 \\
\hline $\mathrm{Z} 4$ & 83.80 & Z47 & 91.72 & Z90 & 89.15 & Z133 & 92.00 & Z176 & 94.10 \\
\hline $\mathrm{Z} 5$ & 86.86 & $\mathrm{Z} 48$ & 99.89 & Z91 & 94.00 & Z134 & 84.50 & Z177 & 88.20 \\
\hline Z6 & 89.56 & Z49 & 98.12 & Z92 & 91.25 & Z135 & 91.12 & Z178 & 87.75 \\
\hline $\mathrm{Z7}$ & 88.24 & $\mathrm{Z} 50$ & 100.00 & Z93 & 90.00 & Z136 & 84.90 & Z179 & 98.98 \\
\hline Z8 & 96.12 & $\mathrm{Z} 51$ & 97.40 & Z94 & 97.14 & Z137 & 81.60 & Z180 & 99.70 \\
\hline Z9 & 93.20 & Z52 & 80.29 & Z95 & 88.86 & Z138 & 93.17 & Z181 & 94.58 \\
\hline $\mathrm{Z} 10$ & 92.22 & Z53 & 98.25 & Z96 & 94.50 & Z139 & 95.10 & Z182 & 98.20 \\
\hline $\mathrm{Z} 11$ & 90.00 & $\mathrm{Z} 54$ & 91.80 & Z97 & 98.57 & $\mathrm{Z} 140$ & 94.00 & $\mathrm{Z} 183$ & 94.58 \\
\hline $\mathrm{Z} 12$ & 98.45 & Z55 & 91.43 & Z98 & 101.59 & Z141 & 105.25 & Z184 & 99.72 \\
\hline Z13 & 96.26 & Z56 & 94.44 & Z99 & 95.25 & Z142 & 97.64 & Z185 & 91.43 \\
\hline Z14 & 100.80 & $\mathrm{Z} 57$ & 94.75 & Z100 & 91.60 & Z143 & 84.57 & Z186 & 96.40 \\
\hline Z15 & 98.50 & Z58 & 84.75 & Z101 & 89.13 & Z144 & 82.20 & Z187 & 95.18 \\
\hline $\mathrm{Z} 16$ & 96.75 & Z59 & 96.00 & Z102 & 97.14 & Z145 & 88.89 & Z188 & 99.20 \\
\hline $\mathrm{Z} 17$ & 112.86 & Z60 & 94.00 & Z103 & 92.00 & Z146 & 86.25 & Z189 & 90.22 \\
\hline Z18 & 96.64 & Z61 & 93.25 & Z104 & 104.29 & Z147 & 88.60 & Z190 & 94.00 \\
\hline Z19 & 92.60 & Z62 & 90.76 & Z105 & 89.00 & Z148 & 90.31 & Z191 & 85.90 \\
\hline $\mathrm{Z} 20$ & 90.76 & Z63 & 90.13 & Z106 & 96.80 & Z149 & 97.14 & Z192 & 84.60 \\
\hline $\mathrm{Z} 21$ & 99.75 & Z64 & 91.00 & Z107 & 94.20 & $\mathrm{Z} 150$ & 85.40 & Z193 & 95.17 \\
\hline $\mathrm{Z} 22$ & 96.60 & Z65 & 90.56 & Z108 & 84.50 & Z151 & 99.43 & Z194 & 93.00 \\
\hline $\mathrm{Z} 23$ & 95.75 & Z66 & 98.50 & Z109 & 99.14 & Z152 & 99.25 & Z195 & 88.57 \\
\hline Z24 & 90.12 & Z67 & 94.68 & Z110 & 96.32 & Z153 & 98.57 & Z196 & 83.20 \\
\hline $\mathrm{Z} 25$ & 100.22 & Z68 & 80.75 & Z111 & 99.74 & Z154 & 101.43 & Z197 & 88.98 \\
\hline Z26 & 92.60 & Z99 & 92.57 & Z112 & 85.80 & Z155 & 98.20 & Z198 & 86.25 \\
\hline $\mathrm{Z} 27$ & 82.76 & $\mathrm{Z70}$ & 96.74 & Z113 & 102.00 & Z156 & 88.16 & Z199 & 88.60 \\
\hline $\mathrm{Z} 28$ & 84.10 & $\mathrm{Z71}$ & 90.00 & Z114 & 100.86 & Z157 & 84.12 & Z200 & 90.31 \\
\hline Z29 & 94.75 & $\mathrm{Z72}$ & 96.32 & Z115 & 93.75 & Z158 & 75.40 & Z201 & 97.14 \\
\hline $\mathrm{Z} 30$ & 96.86 & $\mathrm{Z73}$ & 86.66 & Z116 & 95.00 & Z159 & 104.00 & Z202 & 86.40 \\
\hline Z31 & 106.57 & Z74 & 84.88 & Z117 & 92.66 & $\mathrm{Z} 160$ & 94.25 & Z203 & 99.43 \\
\hline $\mathrm{Z} 32$ & 84.14 & $\mathrm{Z75}$ & 96.76 & Z118 & 90.60 & Z161 & 93.11 & Z204 & 99.25 \\
\hline $\mathrm{Z} 33$ & 87.75 & $\mathrm{Z76}$ & 93.80 & Z119 & 88.30 & Z162 & 100.75 & Z205 & 98.57 \\
\hline Z34 & 90.75 & $\mathrm{Z77}$ & 96.25 & Z120 & 86.00 & Z163 & 89.80 & Z206 & 98.20 \\
\hline $\mathrm{Z} 35$ & 96.13 & $\mathrm{Z78}$ & 94.18 & Z121 & 85.75 & Z164 & 104.29 & Z207 & 88.40 \\
\hline Z36 & 91.11 & Z79 & 93.27 & Z122 & 96.98 & Z165 & 96.90 & Z208 & 94.25 \\
\hline Z37 & 96.50 & $\mathrm{Z} 80$ & 86.45 & Z123 & 96.57 & Z166 & 85.71 & Z209 & 93.11 \\
\hline Z38 & 96.60 & Z81 & 77.60 & Z124 & 92.57 & Z167 & 89.25 & $\mathrm{Z} 210$ & 88.71 \\
\hline Z39 & 93.40 & $\mathrm{Z} 82$ & 87.78 & Z125 & 99.00 & Z168 & 100.61 & $\mathrm{Z} 211$ & 89.25 \\
\hline $\mathrm{Z} 40$ & 102.40 & Z83 & 93.71 & Z126 & 99.71 & Z169 & 87.75 & $\mathrm{Z} 212$ & 87.75 \\
\hline Z41 & 96.29 & Z84 & 99.00 & Z127 & 89.43 & $\mathrm{Z} 170$ & 101.67 & Z213 & 97.67 \\
\hline $\mathrm{Z} 42$ & 96.64 & Z85 & 88.25 & Z128 & 96.00 & $\mathrm{Z} 171$ & 97.67 & & \\
\hline $\mathrm{Z} 43$ & 94.86 & Z86 & 92.25 & Z129 & 86.22 & Z172 & 88.87 & & \\
\hline
\end{tabular}

https://doi.org/10.24264/icams-2020.II.14 

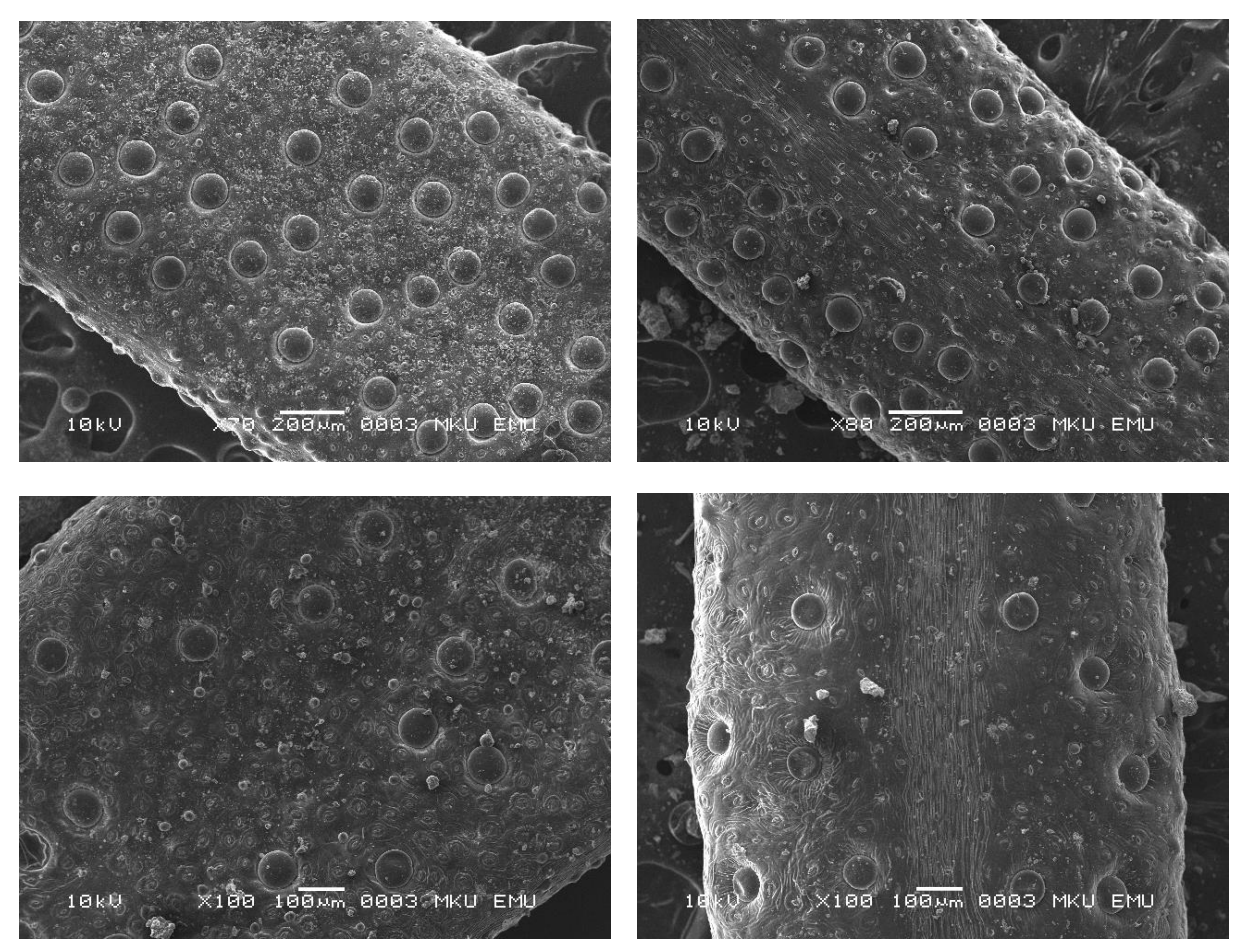

Figure 1. SEM images of some thymbra leaves with the plant code number Z17, Z92, Z144 and Z158, respectively

\section{CONCLUSION}

In the study, first of all, 213 different, well-developed and healthy plants were selected from 68 different locations by single plant selection method. The thymbra plants collected were examined in terms of the number of oil glands per unit area and oil glands diameters in their leaves. It was determined that some plants with low essential oil ratios such as Z144 and Z158 also have low oil grease number and oil gud diameter. It would be appropriate to evaluate these values obtained as preliminary knowledge together with future ontogenetic variability studies.

\section{Acknowledgements}

This study was supported by the Scientific and Technological Research Council of Turkey (TUBITAK-Project No:2140691).

\section{REFERENCES}

Avcı, M. (2005), “Çeşitlilik ve endemizm açısından Türkiye'nin bitki örtüsü”, İstanbul Üniv. Edebiyat Fakültesi Coğrafya Dergisi, (13), 27-55.

Başer, K.H.C. (2002), "Aromatic biodiversity among the flowering plant taxa of Turkey", Pure Appl. Chem., 74(4), 527-545, https://doi.org/10.1351/pac200274040527.

Baydar, H., Sağdiç, O., Özkan, G. and Karadoğan, T. (2004), "Antibacterial activity and composition of essential oils from Origanum, Thymbra and Satureja species with commercial importance in Turkey", Food Control, 15, 169-172, https://doi.org/10.1016/S0956-7135(03)00028-8. 


\section{Oil Glands Number and Oil Glands Diameters of Thymbra spicata var. spicata L. Leaves}

Baytop (1994), Türkçe Bitki Adları Sözlüğü, Ankara: Atatürk Kültür, Dil ve Tarih Yüksek Kurumu Türk Til Kurumu Yayınları, No: 578, P. 168.

Çakan, H. and Byfield, A. (2005), "Amanos dağları" Türkiye'nin 122 önemli bitki alanı (Ed: N.Özhatay, A. Byfield ve S. Atay) WWF Türkiye doğal hayatı koruma vakfi yayını, s:254-257 İstanbul.

Davis, P.H. (1982), Flora of Turkey and the east Aegeans Íslands, Vol. 7. Edinburg University Press.

Doğan, A., Bayrak, A. and Akgül, A. (1987), "Thymol/carvacrol containing Labiatae species and volatile composition of essential oil of Thymbra spicata", Glda, Say1: 6, 359-362.

Ekim, T., Koyuncu, M., Vural, M., Duman, H., Aytaç, Z. and Adıgüzel, N. (2000), Türkiye Bitkileri Kirmızı Kitabı (Ĕgrelti ve Tohumlu Bitkiler), Türkiye Tabiatını Koruma Derneği, Van 100. Yıl Üniv. s:246. Barışcan Ofset. Ankara.

Erken, S. (2005), "Morphological and anatomical studies on Thymbra sintenisii Bornm \&Aznav. (Labiateae)", Turk. J. Bot., 29,389-397.

Hancı Sonsuzer, S., Şahin, S. and Yılmaz, L. (2003), "Isolation of volatile oil from thyme (Thymbra spicata) by steam distillation", Nahrung/Food, 47(4), 252-255, https://doi.org/10.1002/food.200390059.

İnan, M., Kırpık, M., Kaya, D.A. and Kırıcı, S. (2011), "Effect of harvest time on essential oil composition of Thymbra spicata L. Growing in flora of Adiyaman", Advances in Environmental Biology, 5, pp.356-358.

Kizll, S. (2013), "Selection of A clones from Thymbra spicata var. spicata by clonal selection method", Industrial Crops and Products, 41, 1-9, https://doi.org/10.1016/j.indcrop.2012.04.013.

Özcan, M.M., Ünver, A., Uçar, T. and Arslan, D. (2008), "Mineral content of some herbs and herbal teas by infusion and decoction", Food Chemistry, 106, 1120-1127, https://doi.org/10.1016/j.foodchem.2007.07.042.

Özel, M.Z., Göğüş, F. and Lewis, A.C. (2003), "Subcritical water extraction of essential oils from Thymbra spicata", Food chemistry, 82, 381-386, https://doi.org/10.1016/S0308-8146(02)00558-7.

Özgüven, M., Sekin, S., Gürbüz, B., Şekeroğlu, N., Ayanoğlu, F. and Ekren, S. (2005), Tütün, Tıbbi ve Aromatik Bitkiler Üretimi ve Ticareti. Türkiye Ziraat Mühendisliği VI. Teknik Kongresi. 481-501.

Saraç, A. and Tunç, I. (1995), "Residual toxicity and repellency of essential oils to stored-product insets. Rucktandstoxizitat und abstossende wirkung von atherischen ole auf vorratsschadliche insekten", Zeitschrift-fur-Pflanzenkrankheiten und Pflanzenschutz, 102(4), 429-434.

Vural, M. and Aytaç, Z. (2005), "The Flora of Erciyes Dağı (Kayseri, Turkey)”, Tur. J. of Bot., 29, 185-236. 